\title{
Modeling gas and vapor sorption and swelling in a triptycene-based polybenzoxazole: evidence for entropy-driven sorption behavior
}

\author{
Valerio Loianno1,2, Qinnan Zhang 3 , Shuangjiang Luo,, \\ Ruilan Guo ${ }^{3}$ and Michele Galizia ${ }^{*}$ \\ ${ }^{1}$ School of Chemical, Biological and Materials Engineering, University of Oklahoma, 100 E. Boyd Street, \\ Norman 73019, OK, USA \\ ${ }^{2}$ Department of Chemical, Materials and Industrial Production Engineering, University of \\ Naples Federico II, p.le Tecchio 80, 80125 Naples, Italy \\ ${ }^{3}$ Department of Chemical and Biomolecular Engineering, University of Notre Dame, 205 McCourtney \\ Hall, Notre Dame 46556, IN, USA \\ ${ }^{4}$ Beijing Key Laboratory of Ionic Liquids Clean Process, Institute of Process Engineering, Chinese \\ Academy of Sciences, Beijing 100190, China
}

\section{$\underline{\text { SUPPORTING INFORMATION }}$}

*Corresponding author: prof. Michele Galizia

mgalizia@ou.edu, (405) 3255807 
Polymer synthesis, film casting, and thermal treatment. Triptycene dianhydride (TPDAn) monomer was prepared following our previously reported procedure and dried at $160{ }^{\circ} \mathrm{C}$ overnight under vacuum before use [1]. The poly(o-hydroxyimide) copolymer precursor containing $25 \mathrm{~mol} \%$ triptycene unit (TPHI-0.25) was synthesized via polycondensation of TPDAn, 6FDA, and 6FAP in $0.25: 0.75: 1$ molar ratio using solution imidization method, as reported in a previous study [2]. Films of TPHI-0.25 precursor were prepared via solution casting. In a typical case, polymer solution in DMAc ( 7 wt\%) filtered with a $0.45 \mu \mathrm{m}$ Teflon syringe filter was cast onto a leveled glass plate. Films were formed after drying under an infrared lamp $\left(\sim 60^{\circ} \mathrm{C}\right)$ overnight. Following this procedure, the nascent membranes were peeled off and dried at $160{ }^{\circ} \mathrm{C}$ in a vacuum oven overnight to remove any residual solvent. For thermal rearrangement conversion to polybenzoxazole (PBO), precursor films were sandwiched between two porous ceramic plates, and placed in a muffle furnace pre-heated at $300{ }^{\circ} \mathrm{C}$ with high-flow-rate nitrogen purge. After $2 \mathrm{~h}$, the temperature was raised quickly to $450{ }^{\circ} \mathrm{C}$ and maintained for $30 \mathrm{~min}$, after which the film was allowed to slowly cool down to room temperature at a cooling rate no greater than $10{ }^{\circ} \mathrm{C} \mathrm{min}^{-1}$. The final films (TPBO) were $\sim 30-40$ $\mu \mathrm{m}$ thick [2]. 
Structure of HAB-6FDA-TR450-30min

Hereafter, we report the chemical structure of HAB-6FDA-TR450-30min, an iptycene-free TR polymer that is used for the sake of comparison with TPBO throughout this paper.

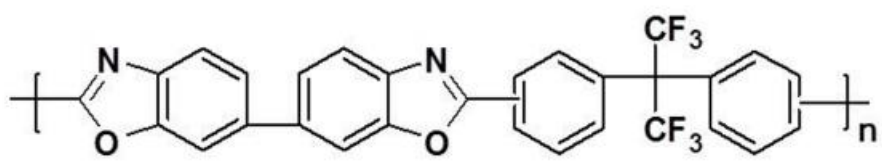

Figure S1. Chemical structure of HAB-6FDA-TR450-30min. 


\section{TPBO swelling upon exposure to $\mathrm{CO}_{2}$ and $\mathrm{C}_{2} \mathrm{H}_{6}$ at multiple temperatures}

$\mathrm{CO}_{2}$ and $\mathrm{C}_{2} \mathrm{H}_{6}$ sorption-induced swelling in TPBO is reported in Figs. S2 A-B as a function of pressure and temperature. At fixed pressure, dilation increases with decreasing temperature, which mirrors the behavior of sorption isotherms.
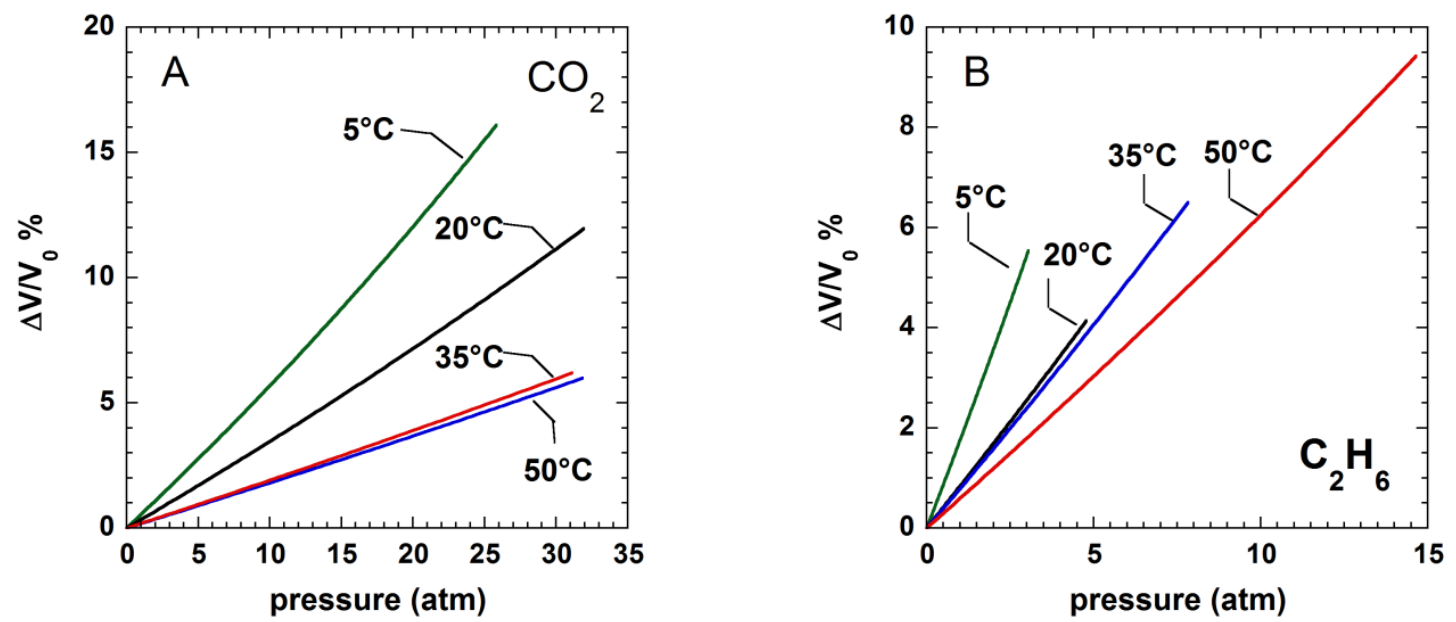

Figure S2. $\mathrm{CO}_{2}(A)$ and $\mathrm{C}_{2} \mathrm{H}_{6}(B)$ induced-swelling of TPBO as a function of pressure and temperature. Swelling isotherms were calculated using the NELF model. 


\section{Ethane partial molar volume in TPBO}
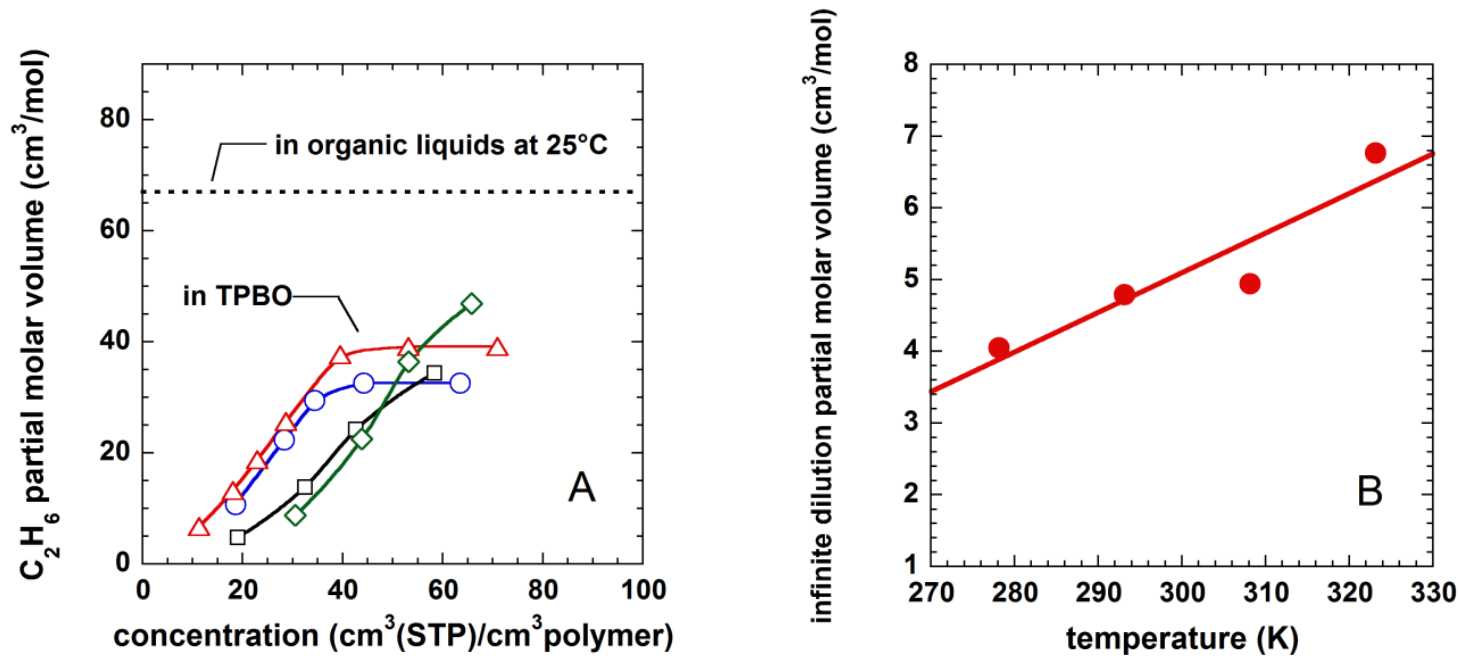

Figure S3. A) Ethane partial molar volume in TPBO as a function of concentration and temperature. Red open triangles: $50^{\circ} \mathrm{C}$; blue open circles: $35^{\circ} \mathrm{C}$; black open squares: $20^{\circ} \mathrm{C}$; green open diamonds: $5^{\circ} \mathrm{C}$. Continuous lines are a guide for the eye. Ethane partial molar volume in organic liquids is from ref. [3]. B) Ethane partial molar volume at infinite dilution as a function of temperature. Continuous line is a linear interpolation.

Ethane partial molar volumes in TPBO at infinite dilution and $35^{\circ} \mathrm{C}$ are lower than $7 \mathrm{~cm}^{3} / \mathrm{mol}$. Ethane partial molar volume in glassy polymers is rarely reported in the literature, therefore it is difficult to put our data in perspective. Merkel et al. measured ethane sorption and dilation in Teflon ${ }^{\circledR}$ AF2400 at $35^{\circ} \mathrm{C}$, from which partial molar volumes were estimated [4]. Ethane partial molar volume in TPBO at infinite dilution and $35^{\circ} \mathrm{C}\left(7 \mathrm{~cm}^{3} / \mathrm{mol}\right)$ is somewhat smaller than that in Teflon ${ }^{\circledR}$ AF2400 at the same temperature $\left(10 \mathrm{~cm}^{3} / \mathrm{mol}\right)$. As discussed in the case of carbon dioxide sorption, this result indicates that TPBO swells less than Teflon ${ }^{\circledR}$ AF2400, which is consistent with the larger free volume exhibited by TPBO relative to Teflon ${ }^{\circledR}$ AF2400 (19.4\% and 
$18 \%$, respectively, based on Eq. 7 in the manuscript), and with the higher rigidity exhibited by the former polymer.

\section{References}

[1] S. Luo, J.R. Wiegand, B. Kazanowska, C.M. Doherty, K. Konstas, A.J. Hill, R. Guo, Finely Tuning the Free Volume Architecture in Iptycene-Containing Polyimides for Highly Selective and Fast Hydrogen Transport. Macromolecules 2016, 49, 3395-3405

[2] S. Luo, Q. Zhang, L. Zhu, H. Lin, B.A. Kazanowska, C.M. Doherty, A.J. Hill, P. Gao, R. Guo, Highly selective and permeable microporous polymer membranes for hydrogen purification and $\mathrm{CO}_{2}$ removal from natural gas. Chem. Mater. 2018, 30, 5322-5332

[3] Y. Kamiya, K. Terada, K. Mizoguchi, Y. Naito, Sorption and partial molar volumes of organic gases in rubbery polymers. Macromolecules 1992, 25, 4321-4324

[4] M.G. De Angelis, T.C. Merkel, V.I. Bondar, B.D. Freeman, F. Doghieri, G.C. Sarti, Gas sorption and dilation in poly(2,2-bistrifluoromethyl-4,5-difluoro-1,3-dioxole-cotetrafluoroethylene): comparison of experimental data with predictions of the non-equilibrium lattice fluid model. Macromolecules 2002, 35, 1276-1288 